Rev Biomed 2006; 17:86-95.

\title{
Coliformes fecales y mesofílicos aerobios en alimentos, superficies y manos del personal y niños de una guardería.
}

\section{Artículo Original}

Jorge Alonzo-Salomón ${ }^{1}$, Mario R. Heredia-Navarrete ${ }^{2}$, Onelia García-Roque ${ }^{3}$

${ }^{1}$ Coordinación de Ingeniería Ambiental, Facultad de Ingeniería, ${ }^{2}$ Centro de Investigaciones Regionales “Dr. Hideyo Noguchi”, Universidad Autónoma de Yucatán, Mérida, Yucatán, México. ${ }^{3}$ Departamento de Estudios de la Contaminación del Instituto Mexicano del Petróleo, Ciudad del Carmen, Campeche, México.

\section{RESUMEN.}

Objetivo. Describir la presencia de coliformes fecales (CF) y mesofílicos aerobios (MA) en alimentos, superficies, manos del personal y de los niños en una guardería y proponer medidas para su control.

Materiales y métodos. Se obtuvieron muestras de los diferentes alimentos que se sirven en una guardería antes de ser consumidas por los niños, de las superficies y de las manos del personal y los niños. Se efectuaron los análisis bacteriológicos para obtener la concentración de CF y MA y se compararon los resultados.

Resultados. Con respecto a los CF en los alimentos, las mayores concentraciones se obtuvieron en la leche con 700 UFC / ml y en la carne con 200 UFC / g; en las superficies las mayores concentraciones se obtuvieron en la mesa 9 UFC / 25 $\mathrm{cm}^{2}$ y en el piso del salón con 4 UFC / $25 \mathrm{~cm}^{2}$. En las manos del personal y de los niños no se encontraron CF.
Con respecto a los MA, en los alimentos, las mayores concentraciones se obtuvieron en la leche, y refresco con 6,700 y 2,800 UFC / ml respectivamente y en la carne con 270 UFC / g; en las superficies las mayores concentraciones se obtuvieron en los biberones, piso y juguetes con 70,000, 400 y 640 UFC / 25 cm², respectivamente. En las manos del personal la mayor concentración de MA fue de 940 UFC / $50 \mathrm{~cm}^{2}$ y en los niños de 860 UFC / $50 \mathrm{~cm}^{2}$.

Conclusiones. La mayor contaminación por CF se presenta en los alimentos, entre los cuales la leche es la fuente principal de contaminación fecal en la guardería, por lo que se sugieren mayores cuidados higiénicos en su preparación, especialmente en el área de los lactantes que son los grupos con los mayores registros de CF en la leche. Con respecto a los MA, la mayor concentración se presenta en los biberones de los lactantes, por lo que asimismo se recomiendan mayores cuidados higiénicos en su limpieza. (Rev Biomed 2006; 17:86-95)

Solicitud de sobretiros: Jorge Alonzo-Salomón, Coordinación de Ingeniería Ambiental, Facultad de Ingeniería, Universidad Autónoma de Yucatán. Apartado Postal 1-150 Cordemex, C.P. 97111, Mérida, Yucatán, México. Recibido el 19/Mayo/2005. Aceptado para publicación el 11/Mayo/2006. Correo electrónico: asalom@uady.mx 


\section{J Alonzo-Salomón, MR Heredia-Navarrete, O García-Roque.}

Palabras clave: coliformes fecales, mesofílicos aerobios, guarderías, contaminación de alimentos.

\section{SUMMARY.}

Faecal coliforms and aerobic mesophiles in food, on surfaces and hands of the personnel and children at a day nursery.

Objective. To describe the presence of fecal coliformes (FC) and aerobic mesophiles (AM) in food, on surfaces and hands of personnel and children at a day-care center and to propose measures for their control.

Materials and methods. At a day-care center, samples were obtained from different foodstuff before being consumed by the children, from the surfaces and from the hands of both the personnel and the children. Bacterial analysis was carried out to determine the concentration of $\mathrm{CF}$ and $\mathrm{AM}$ and the results were compared.

Results. The highest concentrations of FC in food was obtained from milk with $700 \mathrm{UFC/millilitre}$ and from meat with 200 UFC/gram the highest concentrations on surfaces were obtained from the table $9 \mathrm{UFC} / 25 \mathrm{~cm}^{2}$. No FC were found on the hands of neither the personnel nor the children.

The highest concentrations of AM obtained from food was from milk and soft drinks, 6700 and $2800 \mathrm{UFC} /$ millilitre respectively and from meat with 270 UFC/gram; the highest concentrations obtained from surfaces were on the feeding bottles, floor and toys with 70000, 400 and 640 UFC/25 $\mathrm{cm}^{2}$ respectively. The highest concentration of AM found on the hands of personnel was 940 UFC/50 $\mathrm{cm}^{2}$ and $800 \mathrm{UFC} / 50 \mathrm{~cm}^{2}$ on children's hands.

Conclusion. The highest contamination by FC was found in food, among which, milk was the main source of faecal contamination in the daycare center. Hygienic preparation of milk needs to be improved, especially in the area of suckling babies wich is the group with the highest record of FC in milk. As far as AM is concerned, the greatest concentration was found on the feeding bottles of suckling babies, so improved care in the hygenic cleaning of the feeding bottles is also recommendedd. (Rev Biomed 2006; 17:86-95)

Key words: faecal coliformes, aerobic mesophiles, day-care center, contamination of food.

\section{INTRODUCCIÓN.}

El presente trabajo es un estudio sobre la contaminación por coliformes fecales (CF) y mesofilicos aerobios (MA) en una guardería de la ciudad de Mérida, Yucatán, México, y el objetivo es describir su presencia en los alimentos que consumen los infantes, en las superficies con las cuales tienen contacto frecuente, así como en las manos del personal y de los niños. La importancia de identificar las principales fuentes de contaminación bacteriana en la guardería radica en poder proponer y establecer medidas para su control (1-3).

En la actualidad las guarderías han adquirido una importancia vital en nuestra sociedad como centros para el cuidado de los hijos de madres trabajadoras, ya que, al no poder atenderlos en el hogar, utilizan estos servicios para su cuidado. Lo anterior se relaciona con un incremento en el número de madres en la fuerza de trabajo, con un mayor número de guarderías que prestan servicio a la sociedad y con un aumento en el número de niños que son atendidos en las guarderías (4-6). Las guarderías, como centros de cuidado, originan el agrupamiento de infantes y preescolares en ambientes diferentes a los encontrados en el hogar, favoreciéndose las condiciones para que estos niños se vean expuestos a nuevos agentes causantes de enfermedades (7-10). Entre éstas, las diarreicas son una de las principales causas de morbilidad en guarderías (11-13).

Estudios epidemiológicos y microbiológicos ambientales indican que las guarderías pueden favorecer la transmisión de microorganismos causantes de diarrea y otras enfermedades (1417). Frecuentemente los organismos a los que se exponen los infantes son de origen entérico

\section{Revista Biomédica}




\section{Coliformes fecales y mesofílicos aerobios en una guardería.}

y se adquieren por la vía fecal-oral, lo cual da importancia a la materia fecal como un factor que origina la contaminación de alimentos y otros ambientes, resultando en una causa adicional de la presencia de enfermedades diarreicas en guarderías. La falta de higiene del personal que atiende a los niños y de los preparadores de los alimentos, el lavado inadecuado de manos o superficies y de los utensilios, son factores que pueden favorecer que no se eliminen completamente los organismos de origen fecal y éstos puedan contaminar los alimentos que se manejan o consumen y ser una posible causa de diarrea.

Entre los indicadores microbiológicos utilizados para señalar la presencia de contaminación de origen fecal en diversos ambientes, están los $\mathrm{CF}$. Investigaciones realizadas demuestran que es común la presencia de CF en las guarderías (18-19) y que la contaminación con estos microorganismos se relaciona con la presencia de diarrea (18).

Se ha estudiado el impacto de la contaminación microbiana en distintos ambientes de guarderías, y se ha determinado la prevalencia de CF (20), encontrando que es común su presencia en objetos inanimados (área de cambio de pañal, el asa del bote de basura, de los pañales, juguetes, el piso del área de juego y las sillas), y que la contaminación por CF es más frecuente en los salones con niños de 12 a 24 meses de edad que los salones con niños menores de 12 meses (20).

En nuestro medio contamos con información relativa a microorganismos identificados en las heces fecales de los niños enfermos de diarrea (21), sin embargo, no contamos con información sobre CF y MA en diferentes ambientes de la guardería. Estos microorganismos, que amenazan la salud de los niños asistentes, pueden ser trasmitidos por vía alimenticia, objetos inanimados, limpieza inadecuada de las superficies en contacto con los niños, por falta de una higiene adecuada de los manejadores de los alimentos o por la inadecuada limpieza de los utensilios de comida, mesas, etc.

Los datos obtenidos corresponden a dos etapas de la duración del estudio; la primera eta- pa es de febrero de 1995 hasta julio de 1996 (3 semestres), la información recabada durante este periodo es retrospectiva y se obtuvo del análisis de la información contenida en los expedientes de la guardería. La segunda, consistió en el seguimiento prospectivo de una cohorte de niños, realizada de agosto de 1996 hasta julio de 1997 (2 semestres).

El tamaño de la población en estudio es variable con el tiempo, ya que existen tanto entradas (alta en guardería) y salidas (baja en guardería) en cualquier momento en el tiempo, por lo que se tiene una cohorte dinámica, lo que ocasiona que el tamaño de muestra cambie por semestre y grupo de edad (salón).

La población dentro de la guardería esta dividida en grupos de edad (salones). Cada seis meses realizan movimientos de niños hacia otros grupos de mayor edad, los cuales se efectúan en los días finales de los meses de enero y julio. Existe también un periodo de vacaciones entre los meses de julio y agosto, teniendo reinscripciones durante la última semana de agosto. En ocasiones hay nuevos ingresos de niños a la guardería en esos meses, aunque la mayor cantidad de nuevos ingresos es en los meses de febrero y septiembre.

Para la obtención de datos se realizó una revisión de la documentación proporcionada por la guardería y la generada por el seguimiento de la cohorte, todo esto con el fin de generar una base de datos. Los datos de los periodos retrospectivo y prospectivo se obtuvieron de tres fuentes: los expedientes clínicos, las bitácoras de las enfermeras y las listas de control de asistencia de los niños. De los expedientes clínicos se obtuvieron los siguientes datos: Nombre, edad, fecha de nacimiento, padecimientos crónicos, peso y fechas en que se enfermó de diarrea. Las bitácoras se consultaron para obtener el número de casos de diarrea mensual de los niños durante su estancia en la guardería para el periodo retrospectivo. Las niñeras, al tener sospecha de un caso de diarrea, avisaban a las enfermeras. Éstas, de acuerdo a su experiencia, consultaban con el médico de la 
J Alonzo-Salomón, MR Heredia-Navarrete, O García-Roque.

guardería quién era el que finalmente confirmaba (diagnosticaba) o descartaba el caso en cuestión y lo anotaban en las bitácoras y en el expediente clínico del niño. En el periodo prospectivo las bitácoras se consultaban 2 veces por semana para detectar los casos y la fecha de ocurrencia.

Las listas de control de asistencia mensual se utilizaron para obtener las fechas de los movimientos de entrada (alta) y salida (baja) de los niños, ya sea de la guardería o del salón, así como para obtener el tiempo - persona en riesgo por niño.

\section{MATERIAL Y MÉTODOS.}

Condiciones de muestreo: Se realizaron visitas periódicas a la guardería con el propósito de aplicar guías de observación y entrevistas al personal de trabajo para obtener información sobre el funcionamiento de la guardería y definir los puntos de muestreo.

En cada salón se presentaron características propias que se describen a continuación: el salón de lactantes 1 (L1), agrupa infantes de 1.5 a 6 meses de edad; el salón de lactantes 2 (L2), infantes de 7 a 12 meses de edad; el salón de lactantes 3 (L3), infantes de 13 a 18 meses de edad y el salón de maternal 1 (M1), infantes de 19 a 24 meses de edad. Estos salones los consideramos independientes, ya que solamente se encuentran comunicados con el área de cambio de pañal y no existe tránsito de niños entre una y otra área. En el salón de L1, algunos de los infantes permanecen en cunas y "porta bebés", mientras que otros, que están en la llamada etapa del "gateo", permanecen en el piso del salón. En el salón de L2, algunos de los infantes caminan, mientras que otros, que están en la etapa del "gateo", permanecen también en el piso. En el salón de L3, la mayoría de los infantes caminan, mientras que otros, pueden permanecer en el piso del salón durante sus actividades y juegos. En los tres salones de lactantes (L1, L2 y L3) no se permite la entrada con calzado a los niños $\mathrm{y}$ a las personas que transitan en esos ambientes, siendo necesario quitarse los zapatos para entrar y así disminuir el riesgo de contaminación por contacto con el piso.

Además de los salones de lactantes esta el salón de Maternal (M1), en este salón todos los infantes ya caminan, por lo que ellos, las niñeras u otras personas pueden transitar con zapatos en el salón; los infantes de M1 no realizan actividades directamente en el piso, debido a esta situación, al piso de este salón no se le realizó muestreo alguno.

En los cuatro salones estudiados los infantes tienen contacto con diversos juguetes, los cuales son una fuente potencial de contaminación $(20,22)$, ya que frecuentemente se introducen este tipo de objetos en la boca, o bien, contaminan sus manos las cuales también se pueden introducir en la boca. De acuerdo a las características de los salones, el comedor y las personas, se eligió tomar muestras de las superficies, utensilios, alimentos y manos del personal y los niños.

En cuanto a las superficies, en el salón se tomaron muestras del piso y los juguetes; en el comedor, de superficies (mesa) y los utensilios (plato, vaso, biberón y cuchara), y de las personas se tomaron muestras de las manos del personal (maestras, manejadoras de alimentos y los niños). Para los alimentos, se muestrearon dos básicos como son la leche y la carne, pero como estos no eran el único tipo de alimento proporcionado a los niños, se eligió el complemento del día, mencionado en los cuadros 1, 2 y 3 bajo el concepto de frijol, refresco, jugo, tortilla, paleta, gelatina, papilla. Estos complementos variaban de acuerdo al menú del día.

La selección de cada una de las superficies, alimentos y personas fue al azar.

Procedimiento de muestreo: La técnica empleada para la recolección fue de acuerdo al tipo de muestra como se describe a continuación: tanto para las manos como para las superficies inanimadas se utilizó la técnica del hisopo, que consistió en frotar sobre un área determinada $\left(25 \mathrm{~cm}^{2}\right)$ un hisopo estéril humedecido con la solución para recoger la flora microbiana de dicha superficie y después se suspendió en un tubo de

\section{Revista Biomédica}


Coliformes fecales y mesofílicos aerobios en una guardería.

Cuadro 1.

Ambientes examinados y frecuencia de muestras.

\begin{tabular}{|c|c|c|c|}
\hline Ambiente & Ambiente & $\begin{array}{l}\text { Frecuencia de muestras } \\
\text { Positivas para CF (\%) }\end{array}$ & Positivas para MA (\%) \\
\hline $\begin{array}{l}\text { Alimento } \\
\text { Leche } \\
\text { Carne } \\
\text { Frijol } \\
\text { Refresco } \\
\text { Jugơ } \\
\text { Tortilla } \\
\text { Paleta } \\
\text { Gelatina }\end{array}$ & $\begin{array}{l}3 \\
6 \\
1 \\
1 \\
1 \\
1 \\
1 \\
1\end{array}$ & $\begin{array}{l}2(66.7) \\
2(33.3) \\
0(0.0) \\
0(0.0) \\
0(0.0) \\
0(0.0) \\
0(0.0) \\
0(0.0)\end{array}$ & $\begin{array}{l}3(100.0) \\
33(50.0) \\
0(0.0) \\
1(100.0) \\
0(0.0) \\
1(100.0) \\
0(0.0) \\
0(0.0)\end{array}$ \\
\hline Papilla & 1 & $0(0.0)$ & $1(100.0)$ \\
\hline $\begin{array}{l}\text { Superficie } \\
\text { Piso } \\
\text { Juguete } \\
\text { Plato } \\
\text { Mesa } \\
\text { Cuchillo } \\
\text { Jarra } \\
\text { Vaso } \\
\text { Biberón } \\
\text { Cuchara } \\
\text { Tabla de cocina }\end{array}$ & $\begin{array}{l}9 \\
11 \\
2 \\
3 \\
1 \\
1 \\
3 \\
1 \\
1 \\
1\end{array}$ & $\begin{array}{l}2(22.2) \\
0(0.0) \\
0(0.0) \\
2(66.7) \\
0(0.0) \\
0(0.0) \\
0(0.0) \\
0(0.0) \\
0(0.0) \\
0(0.0)\end{array}$ & $\begin{array}{l}9(100.0) \\
11(100.0) \\
2(100.0) \\
2(66.7) \\
1(100.0) \\
1(100.0) \\
2(66.7) \\
1(100.0) \\
0(0.0) \\
1(100.0)\end{array}$ \\
\hline $\begin{array}{l}\text { Manos } \\
\text { Niñeras } \\
\text { Niños } \\
\text { Cocineras }\end{array}$ & $\begin{array}{l}11 \\
4 \\
6\end{array}$ & $\begin{array}{l}0(0.0) \\
0(0.0) \\
0(0.0)\end{array}$ & $\begin{array}{l}10(90.9) \\
4(100.0) \\
6(100.0)\end{array}$ \\
\hline Total & 72 & 8 (11.1) & 59 (81.9) \\
\hline
\end{tabular}

* Jugo Jumex

$\dagger$ Paleta Nestle

ensayo con diluyente (solución salina fisiológica) como medio de transporte para preservar la muestra (en refrigeración a $4^{\circ} \mathrm{C}$ ) a partir del cual se efectuaron los cultivos para los recuentos de unidades formadoras de colonias y su posterior análisis $(23,24)$.

Para la recolecta de la muestra de alimento se utilizaron bolsas estériles cuando el alimento era sólido y en el caso de alimentos líquidos se utilizaron frascos estériles. Una vez recolectada la muestra se conservó en refrigeración $\left(4^{\circ} \mathrm{C}\right)$ y se transportó al laboratorio para su análisis bacteriológico. A todas las muestras se les realizó la búsqueda de CF y MA.

Para conocer el contenido de microorganismos viables de MA se utilizó la técnica cuenta estándar, y para la detección de los CF el método de Mackenzie (23, 24).

Análisis Estadístico: No se le realizaron pruebas de hipótesis a los datos, dado que nuestro interés no era comparar los promedios o totales de las concentraciones de CF o MA en los ambientes muestreados, sino el encontrar la principal fuente de contaminación por CF o MA en la guardería; por tal razón sólo se presentan los resultados en forma de tabla de frecuencias.

\section{RESULTADOS.}

Se tomaron un total de 72 muestras (cuadro 1) de las cuales 18 (25.0\%) fueron de los alimentos, 33 (45.8\%) de las superficies y 21 (29.2\%) de las manos. La contaminación por CF en los alimentos representa el 50.0\% del total de los alimentos positivos para CF, en las superficies representa también el $50.0 \%$ y en las manos del personal y los niños no se encontró contaminación por CF. Asimismo, la contaminación por MA en los alimentos representa el $15.3 \%$ del total, en las superficies representa $50.8 \%$ y en las manos del personal y los niños representa el 33.9\%. 
J Alonzo-Salomón, MR Heredia-Navarrete, O García-Roque.

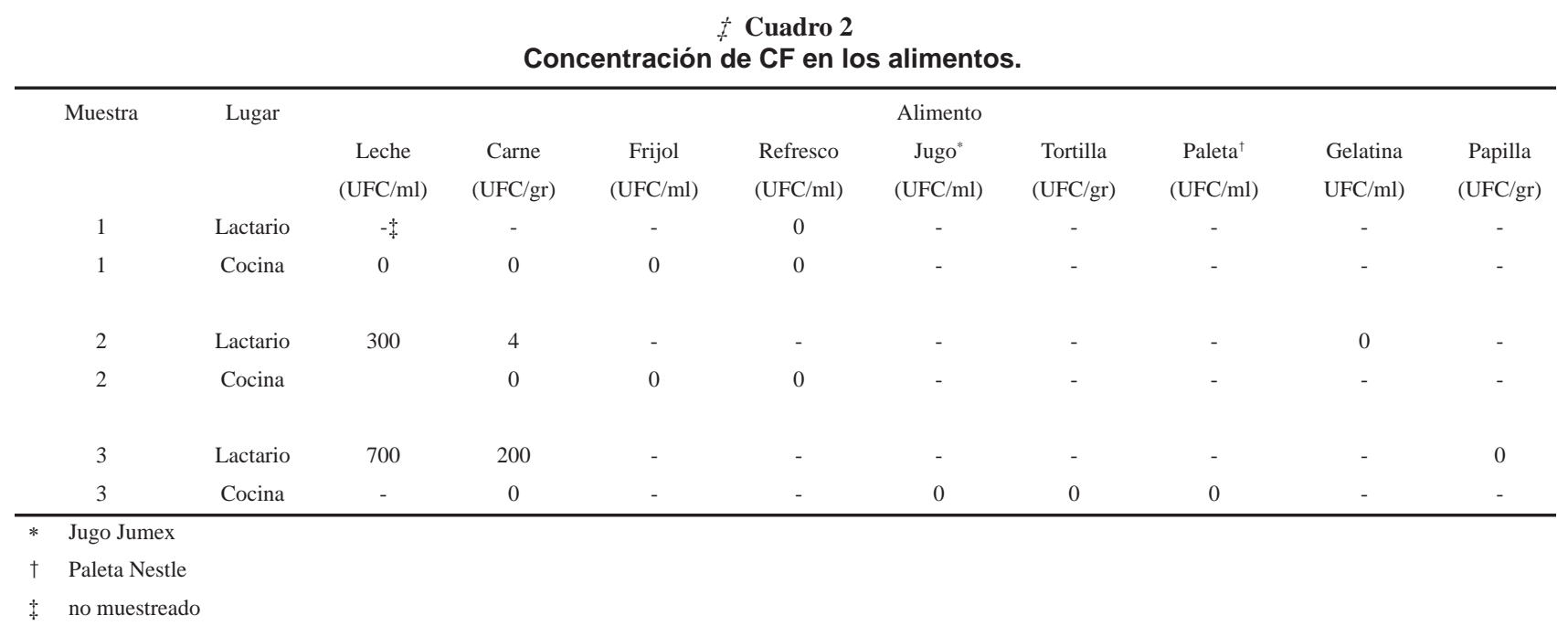

Con respecto a los CF en los distintos ambientes de la guardería (cuadros 2-6), la mayor contaminación por CF en los alimentos se observó en la leche con una concentración de 700 UFC / ml y en la carne del lactario con 200 UFC / g. En las superficies la mayor contaminación por CF se registró en la mesa del comedor con una concentración de 9 UFC / $25 \mathrm{~cm}^{2}$ y en el piso del salón del grupo Lactantes 1 con 4 UFC / 25 cm².

Con respecto a los MA en los distintos ambientes de la guardería (cuadros 2-6), la mayor contaminación por MA en los alimentos se registró en la leche, refresco y papilla con una concentración de 6700, 2800 y 800 UFC / ml, respectivamente, y en la carne del lactario con $270 \mathrm{UFC/} \mathrm{g.}$ En las superficies la mayor concentración de MA se presentó en el biberón con 70,000 UFC / 25 cm², en el piso del grupo Lactantes 2 y en los juguetes del grupo Lactantes 1 con 400 y 640 UFC / 25 $\mathrm{cm}^{2}$, respectivamente; en las manos del personal la mayor concentración de MA fue en las niñeras

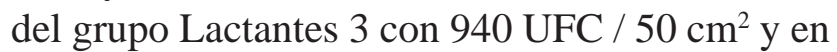
las manos de los niños del grupo Lactantes 3 con $860 \mathrm{UFC} / 50 \mathrm{~cm}^{2}$.

\section{DISCUSIÓN.}

En nuestro estudio encontramos datos sobre la presencia de CF en los alimentos y en las superficies y de MA en todos los estudiados en la guardería (alimentos, superficies y manos). Las muestras positivas para CF en los alimentos fueron obtenidas de la leche y carne y para las

Cuadro 3

Concentración de MA en los alimentos.

\begin{tabular}{|c|c|c|c|c|c|c|c|c|c|c|c|}
\hline & \multirow[t]{2}{*}{ Muestra } & \multirow[t]{2}{*}{ Lugar } & \multicolumn{9}{|c|}{ Alimento } \\
\hline & & & $\begin{array}{c}\text { Leche } \\
\text { (UFC/ml) }\end{array}$ & $\begin{array}{c}\text { Carne } \\
\text { (UFC/gr) }\end{array}$ & $\begin{array}{c}\text { Frijol } \\
\text { (UFC/ml) }\end{array}$ & $\begin{array}{l}\text { Refresco } \\
\text { (UFC/ml) }\end{array}$ & $\begin{array}{c}\text { Jugo }^{*} \\
\text { (UFC/ml) }\end{array}$ & $\begin{array}{c}\text { Tortilla } \\
\text { (UFC/gr) }\end{array}$ & $\begin{array}{c}\text { Paleta }^{\dagger} \\
\text { (UFC/ml) }\end{array}$ & $\begin{array}{l}\text { Gelatina } \\
\text { UFC/ml) }\end{array}$ & $\begin{array}{c}\text { Papilla } \\
\text { (UFC/gr) }\end{array}$ \\
\hline & 1 & Lactario & $-\ddagger$ & - & - & - & - & - & - & - & - \\
\hline & 1 & Cocina & 2200 & 140 & 0 & 0 & - & - & - & - & - \\
\hline & 2 & Lactario & 6700 & 270 & - & - & - & - & - & 0 & - \\
\hline & 2 & Cocina & - & 0 & 0 & 2800 & - & - & - & - & - \\
\hline & 3 & Lactario & 1500 & 200 & - & - & - & - & - & - & 800 \\
\hline & 3 & Cocina & - & 0 & - & - & 0 & 500 & 0 & - & - \\
\hline$*$ & Jugo Jumex & & & & & & & & & & \\
\hline$\dagger$ & Paleta Nestle & & & & & & & & & & \\
\hline$\ddagger$ & no muestreado & & & & & & & & & & \\
\hline
\end{tabular}

\section{Revista Biomédica}




\section{Coliformes fecales y mesofílicos aerobios en una guardería.}

Cuadro 4

Concentración de CF en las superficies.

\begin{tabular}{|c|c|c|c|c|c|c|c|c|c|c|c|}
\hline \multirow{3}{*}{$\begin{array}{c}\text { Muestra } \\
1\end{array}$} & \multirow[t]{2}{*}{ Grupo } & \multicolumn{10}{|c|}{ Superficie } \\
\hline & & Piso & Juguetes & Plato & Mesa & Cuchillo & (UFC/ & 2) & Biberón & Cuchara & Tabla de Cocina \\
\hline & L1 & 0 & 0 & $-\ddagger$ & - & - & - & - & - & - & 1avia - \\
\hline 1 & L2 & 0 & 0 & - & - & - & - & - & - & - & - \\
\hline 1 & L3 & 0 & 0 & - & - & - & - & - & - & - & - \\
\hline 1 & M1 & - & 0 & - & - & - & - & - & - & - & - \\
\hline 1 & Comedor & - & - & 0 & 0 & 0 & - & 0 & - & - & - \\
\hline 1 & Cocina & - & - & - & - & - & - & - & - & - & 0 \\
\hline 1 & Lactario & - & - & 0 & - & - & - & - & - & - & - \\
\hline 2 & L1 & 1 & 0 & - & - & - & - & - & - & - & - \\
\hline 2 & L2 & 0 & 0 & - & - & - & - & - & - & - & - \\
\hline 2 & L3 & 0 & 0 & - & - & - & - & - & - & - & - \\
\hline 2 & M1 & - & 0 & - & - & - & - & - & - & - & - \\
\hline 2 & Comedor & - & - & - & 4 & - & 0 & 0 & - & - & - \\
\hline 2 & Cocina & - & - & - & - & - & - & - & - & - & - \\
\hline 2 & Lactario & - & - & - & - & - & - & - & - & 0 & - \\
\hline 3 & L1 & 4 & 0 & - & - & - & - & - & - & - & - \\
\hline 3 & L2 & 0 & 0 & - & - & - & - & - & - & - & - \\
\hline 3 & L3 & 0 & - & - & - & - & - & - & - & - & - \\
\hline 3 & M1 & - & 0 & - & - & - & - & - & - & - & - \\
\hline 3 & Comedor & - & - & - & 9 & - & - & - & - & - & - \\
\hline 3 & Cocina & - & - & - & - & - & - & 0 & - & - & - \\
\hline 3 & Lactario & - & - & - & - & - & - & - & 0 & - & - \\
\hline
\end{tabular}

$\ddagger \quad$ No muestreado

superficies del piso del salón y mesa del comedor. Lo anterior pone en evidencia la presencia de contaminación fecal tanto en los alimentos que se ofrecen a los niños, así como en áreas donde se alimentan y juegan, por lo que se plantea la necesidad de mejorar las condiciones de higiene en lo que respecta al manejo y preparación de los alimentos y en lo referente a las condiciones de higiene de superficies con las cuales los niños tienen contacto frecuente. No se detectó la presencia de CF en las manos del personal, por lo que se presenta la posibilidad de que los alimentos puedan contaminarse por otro medio que no fueran las manos. Con respecto a los CF en el piso y mesa se observa que en estas superficies las concentraciones son relativamente bajas en comparación a las encontradas en los alimentos.

Para las bacterias MA la mayor contaminación se presenta en las superficies, específicamente en los juguetes y biberón de los lactantes y en la mesa

Cuadro 5

Concentración de Ma en las superficies.

\begin{tabular}{|c|c|c|c|c|c|c|c|c|c|c|c|}
\hline \multirow[t]{2}{*}{ Muestra } & \multirow[t]{2}{*}{ Grupo } & \multicolumn{10}{|c|}{ Superficie } \\
\hline & & Piso & Juguetes & Plato & Mesa & Cuchillo & $\begin{array}{r}\text { (UFC/2 } \\
\text { Jarra }\end{array}$ & Vaso & Biberón & Cuchara & Tabla de Cocina \\
\hline 1 & L1 & 180 & 60 & $-\ddagger$ & & & - & - & & - & \\
\hline 1 & L2 & 400 & 320 & $-{ }^{\top}$ & - & - & - & - & - & - & - \\
\hline 1 & L3 & 180 & 300 & - & - & - & - & - & - & - & - \\
\hline 1 & M1 & - & 280 & & - & - & - & - & - & - & - \\
\hline 1 & Comedor & - & - & 120 & 420 & 100 & - & 200 & - & - & - \\
\hline 1 & Cocina & - & - & - & - & - & - & - & - & - & 200 \\
\hline 1 & Lactario & - & - & 90 & - & - & - & - & - & - & - \\
\hline 2 & L1 & 40 & 4 & - & - & - & - & - & - & - & - \\
\hline 2 & L2 & 86 & 45 & - & - & - & - & - & - & - & - \\
\hline 2 & $\mathrm{~L} 3$ & 22 & 3 & - & - & - & - & - & - & - & - \\
\hline 2 & M1 & - & 3 & - & - & - & - & - & - & - & - \\
\hline 2 & Comedor & - & - & - & 18 & - & 2 & 20 & - & - & - \\
\hline 2 & Cocina & - & - & - & - & - & - & - & - & - & - \\
\hline 2 & Lactario & - & . & & & & 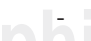 & 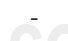 & - & 0 & - \\
\hline 3 & L1 & 160 & 640 & - & - & - & - & - & - & - & - \\
\hline 3 & L2 & 360 & 70 & - & - & - & - & - & - & - & - \\
\hline 3 & $\mathrm{~L} 3$ & 390 & - & - & - & - & - & - & - & - & - \\
\hline 3 & M1 & - & 150 & - & - & - & - & - & - & - & - \\
\hline 3 & Comedor & - & - & - & 530 & - & - & - & - & - & - \\
\hline 3 & Cocina & - & - & - & - & - & - & 0 & - & - & - \\
\hline 3 & Lactario & - & - & - & - & - & - & - & 70000 & - & - \\
\hline
\end{tabular}


J Alonzo-Salomón, MR Heredia-Navarrete, O García-Roque.

Cuadro 6

Concentración de CF y MA en manos del personal y niños.

\begin{tabular}{|c|c|c|c|c|c|}
\hline \multirow[t]{2}{*}{ Muestra } & \multirow[t]{2}{*}{ Grupo } & \multicolumn{2}{|c|}{ CF en manos } & \multicolumn{2}{|c|}{ MA en manos } \\
\hline & & Personal & Niños & Personal & Niños \\
\hline & & (UFC/50ml) & (UFC/50 ml) & (UFC/50ml) & (UFC/50 ml) \\
\hline 1 & L1 & 0 & $-\ddagger$ & 620 & - \\
\hline 1 & L2 & 0 & - & 450 & - \\
\hline 1 & L3 & 0 & - & 830 & - \\
\hline 1 & M1 & 0 & - & 940 & - \\
\hline 1 & Cocina & 0 & - & 380 & - \\
\hline 1 & Lactario & 0 & - & 400 & - \\
\hline 2 & L1 & 0 & - & 60 & - \\
\hline 2 & L2 & 0 & - & 26 & - \\
\hline 2 & L3 & 0 & - & 45 & - \\
\hline 2 & M1 & 0 & - & 13 & - \\
\hline 2 & Cocina & 0 & - & 500 & - \\
\hline 2 & Lactario & 0 & - & 800 & - \\
\hline 3 & L1 & 0 & 0 & 60 & 660 \\
\hline 3 & L2 & 0 & 0 & 320 & 160 \\
\hline 3 & L3 & 0 & 0 & - & 860 \\
\hline 3 & M1 & 0 & 0 & 0 & 780 \\
\hline 3 & Cocina & 0 & - & 140 & - \\
\hline 3 & Lactario & 0 & - & 70 & - \\
\hline
\end{tabular}

del comedor, las concentraciones observadas en los tres casos son altas, con una concentración muy por encima en los biberones. Con respecto a las manos del personal y los niños, se tienen también concentraciones altas. En los alimentos se observa una fuerte contaminación por MA en la leche del lactario, y en una menor concentración en la carne. Las concentraciones de MA observadas en otros puntos de la guardería tales como el cuchillo, plato, vaso, tabla de cocina, carne de lactario son relativamente bajas.

\section{CONCLUSIONES.}

La mayor contaminación por CF se presenta en los alimentos, entre los cuales la leche es la fuente principal de contaminación fecal en la guardería, por lo que se sugieren mayores cuidados higiénicos en su preparación, especialmente en el área de los lactantes que son los grupos con los mayores registros de CF en la leche. Con respecto a los MA, la mayor concentración se presenta en los biberones de los lactantes, por lo que asimismo se recomiendan mayores cuidados higiénicos en su limpieza.

Las enfermedades de transmisión alimentaria y los daños provocados por los alimentos son, en el mejor de los casos, desagradables, y en el peor pueden ser fatales. Por lo tanto, todas las personas tienen derecho a esperar que los alimentos que comen sean inocuos y aptos para el consumo. Por consiguiente, es imprescindible implementar un control eficaz de la higiene en la guardería, a fin de evitar las consecuencias perjudiciales que derivan de las enfermedades y los daños provocados por los alimentos y por el deterioro de los mismos - como se demuestra en este estudio a través de los resultados encontrados-, para la salud de los niños.

Se debe tener en cuenta que los hábitos de consumo de los alimentos han sufrido cambios importantes en muchos países durante los dos últimos decenios, en consecuencia, se han perfeccionado nuevas técnicas en la preparación y el manejo de los alimentos. Por lo tanto, los responsables de la preparación o elaboración de alimentos, deben examinar el contenido de los documentos donde se exponen las técnicas para la preparación higiénica y en su caso, deberán implementarlas durante su labor.

Por lo expuesto anteriormente, creemos que

\section{Revista Biomédica}




\section{Coliformes fecales y mesofílicos aerobios en una guardería.}

las políticas de las guarderías deberán tener en cuenta la vulnerabilidad de los niños a los procesos infecciosos y en este sentido, deben buscar la mejor manera de fomentar la aplicación de los principios generales de calidad higiénica en la preparación de los alimentos. Para ello, se sugiere a las guarderías, que al momento de diseñar sus programas de salud, tengan en cuenta los puntos siguientes:

- Se deberá proteger adecuadamente a los niños, de las enfermedades o daños causados por los alimentos.

- Se garantizará que durante la elaboración de los alimentos, se de cumplimiento a las normas higiénicas establecidas para esos procesos.

- Deberán establecer programas de educación en materia de salud, que permitan capacitar eficazmente a los preparadores o manipuladores de los alimentos en los principios de calidad higiénica.

- La guardería en su conjunto, deberá aplicar las prácticas de higiene establecidas en la Norma Oficial Mexicana a fin de proporcionar alimentos que sean inocuos y aptos para el consumo.

- Asegurar que los preparadores de alimentos dispongan de una información clara y fácil de comprender -mediante el etiquetado y otros medios apropiados-, la manera en que pueden proteger sus alimentos de la contaminación y del desarrollo o supervivencia de patógenos, almacenándolos, manipulándolos y preparándolos correctamente.

\section{REFERENCIAS}

1.- Reynolds KA, Watt PM, Boone SA, Gerba CP._Occurrence of bacteria and biochemical markers on public surfaces. Int J Environ Health Res 2005; 15:225-34.

2.- Brady MT. Infectious disease in pediatric out-of-home child care. Am J Infect Control 2005; 33:276-85.

3.- Holt J, Skifte TB, Koch. A. Infection control in day-care centers in Greenland. Int J Circumpolar Health 2004; 63 Suppl 2:256-60.

4.- Peth R. The NICHD Study of Early Child Care. Public Information and Communication Branch of the National
Institute of Child and Human Development. Documento disponible en: URL: http://www.nichd.nih.gov/publications/ pubs/early_child_care.htm

5.- Baqui, AH, Black RE, Sack RB, Yunus MD, Siddique AK. Infectious diarrhea in children: controlling transmission in the child care setting. J Paediatr Child Health 1994; 30: 201-9.

6.- Holmes SJ, Morrow AL, Pickering LK. Child-care practices: effects of social change on the epidemiology of infectious diseases and antibiotic resistance. Epidemiol Rev 1996; 18: 10-28.

7.- Barros H, Lunet N. Association between child-care and acute diarrhea: a study in Portuguese children. Rev Saude Publica 2003; 37:603-8.

8.- Cozad A, Jones RD. Disinfection and the prevention of infectious disease. Am J Infect Control 2003; 31:243-54.

9.- Reeves RR, Morrow AL, Bartlett AV, Caruso CJ, Plumb $\mathrm{RL}$, Bening T, et. al. Child day care increases the risk of clinic visits for acute diarrhea and diarrhea due to rotavirus. Am J Epidemiol 1993; 137:97-107.

10.- Churchill RB, Pickering LK. Infection control challenges in child-care centers. Infect Dis Clin North Am 1997; 11: 347-65.

11.- Wald ER, Dashefsky B, Byers C, Guerra N, Taylor F. Frequency and severity of infections in day care. J Pediatr 1988; 112: 540-6.

12.- Bartlett AV, Moore M, Gary GW, Karen M S, Erben JJ, Betty AM. Diarrheal illness among infants and toddlers in day care centers. I Epidemiology and pathogens. Pediatrics 1985; 107: 495-502.

13.- Pickering LK, Evans DG, DuPont HL, Volled JI, Evans DJ. Diarrhea caused by Shigella, Rotavirus, and Giardia in day-care centers: prospective study. J Pediatr 1981; 99: 51-6

14.- Reves RR, Morrow AL, Bartlett AV, Caruso CJ, Plumb RL, Bening T, et. al. Child day care increases the risk of clinic visits for acute diarrhea and diarrhea due to rotavirus. Am J Epidemiol 1993; 137: 97-107.

15.- Van R, Wun CC, Morrow AL, Pickering LK. The effect of diaper type and over clothing on fecal contamination in day-care centers. JAMA 1990; 265: 1840-4 


\section{J Alonzo-Salomón, MR Heredia-Navarrete, O García-Roque.}

16.- Van R, Morrow AL, Reves RR, Pickering LA. Environmental contamination in child day-care centers. Am J Epidemiol 1991; 133: 460-70.

17.- Weniger BG, Ruttenberg AJ, Goodman RA, Juranek DD, Wahlquist SP, Smith D. Fecal coliforms on environmental surfaces in two day care centers. Appl Environ Microbiol 1983; 45: 733-5.

18.- Ekanem EE, DuPont HL, Pickering LK, Selwyn B, Hawkins CM. Transmission dynamics of enteric bacteria in day care centers. Am J Epidemiol 1983;118:562-72.

19.- Weniger BC, Ruttenber AJ, Goodman RA, Juranek DD, Wahlquist SP, Smith D. Fecal coliforms on environmental surfaces in two day-care centers. Appl Environ Microbiol 1983;45:733-5.

20.- Laborde DJ, Weigle KA, Weber DJ, Kotch JB. Effect of fecal contamination in diarrheal illness rates in day-care centers. Am J Epidemiol 1993; 138:243-55.

21.- Oberhelman RA, Flores-Abuxapqui J, Suárez-Hoil G, Puc-Franco M, Heredia-Navarrete M, Vivas-Rosel M, et al, L Gutiérrez-Cogco. Asymptomatic salmonellosis among children in day-care centers in Mérida, Yucatán, México. Pediatr Infect Dis J 2001; 20:792-7.

22.- Alonzo-Salomon J, García-Roque O, Heredia-Navarrete $\mathrm{R}$. Time of attendance and diarrea incidence in infants who attended a day care center. Rev Biomed 2003; 14:153-7.

23.- American Public Health Association (APHA). Compendium of methods for the microbiological examination of foods. Washington (DC); 1976.

24.- Fernández-Escartín E. Microbiología Sanitaria. Vol 1. Universidad de Guadalajara. ANUIES-SEP; 1981.

\section{Revista Biomédica}

